FFIA-UV/04-05

\title{
Supertubes versus superconducting tubes
}

\author{
Rubén Cordero $^{a *}$ and Efraín $\operatorname{Rojas}^{b \dagger}$ \\ a Departamento de Física, Escuela Superior de Física y Matemáticas del I.P.N. \\ Unidad Adolfo López Mateos, Edificio 9, 07738 México, D.F., MEXICO \\ ${ }^{b}$ Facultad de Física e Inteligencia Artificial, Universidad Veracruzana \\ Sebastián Camacho 5, Xalapa, Veracruz; 91000, MEXICO
}

July 18, 2018

\begin{abstract}
In this paper we show the relationship between cylindrical D2-branes and cylindrical superconducting membranes described by a generic effective action at the bosonic level. In the first case the extended objects considered, arose as blown up type IIA superstrings to D2-branes, named supertubes. In the second one, the cosmological objects arose from some sort of field theories. The Dirac-Born-Infeld action describing supertubes is shown to be equivalent to the generic effective action describing superconducting membranes via a special transformation.
\end{abstract}

(Dedicated to Prof. Alberto García on the ocassion of his 60th birthday)

*Email: cordero@esfm.ipn.mx

${ }^{\dagger}$ Email: efrojas@uv.mx 


\section{Introduction}

Nowadays, a number of outstanding problems in physics are currently being worked out using techniques involving the string theory machinery. Every time new ideas transform our understanding of string theory. Since their appeareance, the so-called $D p$-branes $[1,2]$ paved the way for a fierce study in string theory because is assumed that they are source for the Ramond-Ramond fields in Type II theories besides of be a useful tool to explain several supersymmetric and non-supersymmetric field theories. Among main

features of $D p$-branes, resides that 10-dimensional superstrings can end on them. The incorporation of $D p$-branes in superstring picture gives a lot of issues in the theory of solitonic states in nonperturbative string theory and permits to reveal different aspects of string/ $M$-theory dualities.

Recently, special D2-branes named supertubes, have emerged as worldvolume realizations of some kind of sigma models [3, 4], exhibiting more features for the intringuing $M$-theory. The role are playing Born-Infeld fields describing cylindrical D2-branes is to maintain stability against tension, including supergravity forces. On other hand, at cosmological context, some field theories predict the existence of topological defects like strings or domain walls holding ability of carry some kind of charge $[5,6,7,8,9,10]$. For these superconducting strings/membranes, a scalar field living onto the worldsheet plays a similar role as before, i.e., maintain stability against tension. Due to previous similarities, it is to be expected similar descriptions in their dynamics, i.e., must exists a transformation between both dynamical descriptions.

In this paper we shall concentrate on the dynamical similarities between supertubes and superconducting cylindrical branes which are extended objects arose at first instants of the universe. The paper is organized as follows. In Sect. 2 we explain the notation we use through the paper and obtain the canonical momenta associated to the embedding variables by means of variational techniques and with the help of Noether theorem $[11,12]$, in order to identify the energy density. In Sect. 3 we describe the main results about supertubes developed in [3]. In Sect. 4 we write the generic effective action which provide us the dynamics of superconducting extended objects. The comparison of both dynamics is given in Sect. 5. Finally we end the paper with some comments.

\section{Noether currents and momenta}

We consider a D $p$-brane of dimension $d$ evolving in a $N+1$ dimensional background spacetime with metric $g_{\mu \nu}, \mu, \nu=0,1 \ldots, N$. The D $p$-brane worldvolume is an oriented 
timelike manifold of dimension $d$, usually denoted by $m$, endowed with an induced metric $\gamma_{a b}$ from the bulk. If $x^{\mu}=X^{\mu}\left(\xi^{a}\right)$ are the embedding functions of the $\mathrm{D} p$-brane, the induced metric on the worldvolume is given by $\gamma_{a b}=g_{\mu \nu} X^{\mu}{ }_{a} X^{\nu}{ }_{b}$. $\xi^{a}$ denotes the worldvolume coordinates.

We demand that the Dirac-Born-Infeld action (DBI) specify the motion of the $D p$ brane,

$$
S_{B I}=\alpha \int_{m} d^{d+1} \xi \sqrt{-\operatorname{det}\left(\gamma_{a b}+F_{a b}\right)}
$$

where $\alpha$ is the tension of the D $p$-brane, $F_{a b}=2 \partial_{[a} A_{b]}$ is the electromagnetic tensor associated to the worldvolume $U(1)$ gauge field $A_{a} ; a, b=0,1, \ldots, d$.

A general variation of the action (2.1) always cast out in the form $[11,12]$

$$
\begin{aligned}
\delta S_{B I} & =\int_{m}\left\{\mathcal{E}_{\mu} \delta X^{\mu}+\mathcal{E}^{a} \delta A_{a}+\sqrt{-\gamma} \nabla_{a} F^{a}[\delta X ; \delta A]\right\}, \\
& =\int_{m}\left\{\mathcal{E}_{\mu} \delta X^{\mu}+\mathcal{E}^{a} \delta A_{a}\right\}+\int_{\partial m}\left\{P_{\mu} \delta X^{\mu}+\pi^{a} \delta A_{a}\right\},
\end{aligned}
$$

where $\mathcal{E}_{\mu}$ and $\mathcal{E}^{a}$ are the Euler Lagrange derivatives of $\mathcal{L}_{B I}$ with respect to $X^{\mu}$ and $A_{a}$, respectively; $F^{a}$ is an operator defined on the worldvolume and it is related to the momenta associated to the configuration space. The argument of the $F^{a}$, is indicated within the square bracket. $P_{\mu}$ and $\pi^{a}$ are canonical momenta associated to the configuration space. We are interested in the momentum $P_{\mu}$ given by

$$
P_{\mu}=\sqrt{h} T^{a b} \eta_{a} X_{\mu, b},
$$

where $T^{a b}$ is the energy-momentum tensor (see (2.7)) and $\eta_{a}$ is the unit timelike normal vector on the boundary $\partial m$. In fact, when one perform a foliation of the worldvolume in spacelike hypersurfaces $\Sigma$, the corresponding normal vector is $\eta^{a}$.

When the classical equations of motion are satisfied, $\mathcal{E}_{\mu}=0$ and $\mathcal{E}^{a}=0$, these equations are equivalent to the set

$$
\begin{aligned}
T^{a b} K_{a b}^{i} & =0, \\
\nabla_{a} T^{a b} & =0, \\
\nabla_{a} \mathcal{J}^{a b} & =0,
\end{aligned}
$$

where $T^{a b}$ denotes the symmetric energy-momentum tensor, $\mathcal{J}^{a b}$ denotes an antisymmetric bicurrent density and $K_{a b}^{i}$ denotes the extrinsic curvature of the worldvolume, where $i$ runs from 1 to $N+1-d$, i.e., $i$ labels the number of unit normals to the worldvolume. Explicitly, $T^{a b}$ and $\mathcal{J}^{a b}$ are given by

$$
\begin{aligned}
T^{a b} & =\frac{\sqrt{-M}}{\sqrt{-\gamma}}\left(M^{-1}\right)^{(a b)}, \\
\mathcal{J}^{a b} & =\alpha \sqrt{-M}\left(M^{-1}\right)^{[a b]},
\end{aligned}
$$


where $\left(M^{-1}\right)^{a b}$ means the inverse matrix of $M_{a b}:=\gamma_{a b}+F_{a b}$ and $M=\operatorname{det}\left(M_{a b}\right)$.

In order to illustrate the previous results, from (2.1), the corresponding Lagrangian density for $D 2$-branes is

$$
\mathcal{L}=\sqrt{-\gamma}\left(1+\frac{1}{2} F_{a b} F^{a b}\right)^{1 / 2}
$$

where $\gamma$ denotes the determinant of $\gamma_{a b}$. In such case, we are able to write explicitly both the symmetric and antisymmetric parts of the inverse matrix of $M_{a b}$, namely

$$
\begin{aligned}
& \left(M^{-1}\right)^{(a b)}=\gamma^{a b}-\left(1+F^{2}\right)^{-1 / 2} F^{c(a} F_{c}^{b)} \\
& \left(M^{-1}\right)^{[a b]}=-\left(1+F^{2}\right)^{-1 / 2} F^{a b}
\end{aligned}
$$

where we use the notation $F^{2}=(1 / 2) F_{a b} F^{a b}$.

\section{Supertubes}

Brane expansion is an interesting effect in physics. A current topic in $D p$-brane physics concerns expansions from one configuration to another. Interaction of $D p$-branes with external fields (not necessarily at supersymmetric level), under certain circumstances, produce expansions in order to stabilize a given configuration. It has been recently shown [3] that type IIA strings can blow up to produce cylindrical $D 2$-branes, being the angular momentum the stabilizer for maintaining a finite radius for the cylinder.

Following [3], consider a static tubular D2-brane with radius $R(z, \phi)$, embbeded in a $N=10$ Minkowski spacetime

$$
d s^{2}=-d T^{2}+d Z^{2}+d R^{2}+R^{2} d \Phi^{2}+d s^{2}\left(E^{6}\right)
$$

with worldvolumen coordinates $T=t, Z=z, \Phi=\phi$, where the axis is along the $z$ direction. The induced metric is given by

$$
d s^{2}=-d t^{2}+d z^{2}+R^{2} d \phi^{2}+\left(\partial_{z} R d z+\partial_{\phi} R d \phi\right)^{2} .
$$

Assuming time independent Born-Infeld fields via the 2-form field strength

$$
F=E d t \wedge d z+B d z \wedge d \phi
$$

the D2-brane Born-Infeld Lagrangian (2.1) is

$$
\mathcal{L}=\sqrt{\left(R^{2}+R_{\phi}^{2}\right)\left(1-E^{2}\right)+B^{2}+R^{2} R_{z}^{2}},
$$


where $R_{\phi}=\partial_{\phi} R$ and $R_{z}=\partial_{z} R$. Defining the electric displacement $\Pi \equiv \partial \mathcal{L} / \partial E$, the associated Hamiltonian density to (3.15) results

$$
\mathcal{H}=\Pi E-\mathcal{L}
$$

There is a relation between the electric field and the electric displacement given by

$$
E=\frac{\Pi}{R} \sqrt{\frac{B^{2}+R^{2}}{\Pi^{2}+R^{2}}},
$$

such in the case when $R$ is constant and the energy density becomes

$$
\mathcal{E}=\frac{1}{R} \sqrt{\left(\Pi^{2}+R^{2}\right)\left(B^{2}+R^{2}\right)} .
$$

The $z$ independence of the electric displacement comes from the fact that of $\Pi$ is subject to the Gauss law constraint.

\section{Superconducting tubes}

To describe superconducting branes we consider the effective action $[5,6,7,8,9,10]$

$$
S_{c}=\int_{m} d^{d+1} \xi \sqrt{-\gamma} L(\omega),
$$

where $L(\omega)$ denotes a specific model depending on internal fields acting on the brane through the combination $\omega:=\gamma^{a b} \phi_{, a} \phi_{, b}$. There is an important model developed by Nielsen [13], which consists basically in consider a scalar field living on the worldvolume which, under certain circumstances, prevents the collapse. For the case of a superconducting membrane, this model is given by

$$
L=\sqrt{k_{1}+k_{2} \omega}
$$

where $k_{1}$ and $k_{2}$ are constants. The corresponding equations of motion are (compare with (2.4) and (2.6))

$$
\begin{aligned}
T^{a b} K_{a b}^{i} & =0, \\
\nabla_{a} J^{a} & =0,
\end{aligned}
$$

where the explicit form of the energy-momentum tensor is

$$
T^{a b}=L(\omega) \gamma^{a b}-2 \frac{d L}{d \omega} \gamma^{a c} \gamma^{b d} \phi_{, c} \phi_{, d}
$$

Note that the second equation (4.22) corresponds to a conservation law being the conserved current defined as $J^{a}=2 \frac{d L}{d \omega} \gamma^{a b} \nabla_{b} \phi$. 
We assume a background 4-dimensional Minkowski metric of the form

$$
d s^{2}=-d t^{2}+d r^{2}+r^{2} d \theta^{2}+d z^{2},
$$

and we specialize to a superconducting tube described by the embedding

$$
x^{\mu}(t, \theta, z)=\left(\begin{array}{c}
t \\
r(t) \\
\theta \\
z
\end{array}\right),
$$

where the radius $r=r(t)$ is $\theta$ and $z$ independent. The induced metric on the tube is given by

$$
d s^{2}=\left(-1+\dot{r}^{2}\right) d t^{2}+r^{2} d \theta^{2}+d z^{2} .
$$

Under the following assumption for the scalar field, $\phi=\phi_{1}(t)+N \theta$, and from the conservation Eq. (4.22) we get how evolve in time the scalar field

$$
\dot{\phi}=-\frac{\Omega \sqrt{1-\dot{r}^{2}}}{2 r(d L / d \omega)},
$$

where $\Omega$ is an integration constant and $N$ is a constant to be determined. It is straightforward to obtain the form of $\omega$

$$
\omega=\frac{N^{2}-\left(\Omega^{2} k_{1} / k_{2}\right)^{2}}{r^{2}+\left(\Omega^{2} / k_{2}\right)} .
$$

Handling the previous results allow us to write the explicit dependence of the Lagrangian (4.20),

$$
L=\sqrt{\frac{k_{1} r^{2}+k_{2} N^{2}}{r^{2}+\left(\Omega^{2} / k_{2}\right)}} .
$$

\section{Mechanical equivalence between tubes}

Now we turn to show the similarity in dynamics between supertubes and superconducting tubes. When $R$ is $\theta$ and $\phi$ independent, this fact allows us to rewrite the Lagrangian as

$$
\mathcal{L}=-\sqrt{R^{2}\left(1-E^{2}\right)+B^{2}} .
$$

The constant $N$ appearing in the definition of the scalar field, can be computed in terms of $\Pi$ using the relation between (4.22) and (2.5). Inserting the results for $L(\omega)$ and $d L / d \omega$ in the definition of the electric displacement, we get $\Pi=\left(k_{2} / k_{1}\right)^{1 / 2} N$. 
We look at the zero-zero component in the energy-momentum tensor

$$
\begin{aligned}
T^{00} & =L(\omega) \gamma^{00}-2 \frac{d L}{d \omega}\left(\gamma^{00}\right)^{2} \dot{\phi}^{2} \\
& =\frac{1}{r^{2}\left(-1+\dot{r}^{2}\right)} \sqrt{\left(k_{1} r^{2}+k_{2} N^{2}\right)\left(k_{2} r^{2}+\Omega^{2}\right)}
\end{aligned}
$$

such that, for the static configuration $r=R$ the energy density can be extracted from the time component of the momentum vector (2.3), i.e.,

$$
\begin{aligned}
\mathcal{E} & =\frac{1}{R} \sqrt{\left(k_{1} R^{2}+k_{2} N^{2}\right)\left(k_{2} R^{2}+\Omega^{2}\right)} \\
& =\frac{1}{R} \sqrt{k_{1}\left(R^{2}+\pi^{2}\right)\left(k_{2} R^{2}+B^{2}\right)}
\end{aligned}
$$

If we choose $B=\Omega$ and $k_{1}=k_{2}=1$ then $\Pi=N$ and we get the energy in accordance with the result arose from Born-Infeld action (3.18), specifically for the case of supertubes [3]. It is well known $[5,8,10]$ that equilibrium configurations for superconducting extended objects are reached thanks to the current produced by the scalar field, avoiding the collapse. Actually, $\phi$ plays a similar role as the angular momentum for the supertubes case.

\section{Concluding remarks}

We have shown an equivalence, at the bosonic level, between supertubes and superconducting tubes. Superconducting membranes are described uniquely by a scalar field living on its worldvolume. The energy in both tube cases turns out to be same if we relate the conserved type IIA string charge and the D0-brane charge per unit length carried by the tube with the current and the charge of the superconducting tube, respectively. The next task is to explore the possibility to introduce supersymmetry in the superconducting membranes approach and show explicitly its character $1 / 4$ supersymmetric in order to complete the proof of total equivalence between supertubes and superconducting tubes. The last issue is under current investigation.

\section{Acknowledgments}

ER and RC received partial support from CONACyT grant CO1-41639. ER acknowledges also partial support from PROMEP 2003-2004. RC thanks COFAA and CGPI grant 20030642 as well as SNI-CONACyT for financial support. 


\section{REFERENCES}

[1] G. W. Gibbons, Nucl. Phys. B 514603 (1998)

[2] J. Polchinski, TASI Lectures on D-branes hep-th/9611050

[3] D. Mateos and Paul K. Townsend, Phys. Rev. Lett. 87011602 (2001)

[4] D. Mateos, Selena Ng and Paul K. Townsend, JHEP 02003:016 (2002)

[5] A. Vilenkin and E.P.S. Shellard, Cosmic String and Other Topological Defects Cambridge Univ. Press, Cambridge 1994

[6] G. Lazarides and Q. Shafi, Phys. Lett. B 152261 (1985)

[7] P. Peter, J. Phys. A 295125 (1996)

[8] R. Cordero and E. Rojas, Phys. Lett. B 47045 (1999)

[9] A. L. Larsen, Class. Quant. Grav. 101541 (1993)

[10] B. Carter, Brane Dynamics for Treatment of Cosmic Strings and Vortons Lectures given at the Second Mexican School on Gravitation and Physical Mathematics, Tlaxcala, México, hep-th/9705172

[11] R. Capovilla and J. Guven, Phys. Rev. D 6736 (1995)

[12] G. Arreaga, R. Capovilla and J. Guven, Annals of Phys. 279126 (2000)

[13] N. Nielsen, Nucl. Phys. B 167249 (1980) 